\title{
Survey of synthesis and application of Molybdenum Diselenide Shuyan $\mathrm{Qi}^{\mathrm{a}}$, Xiaohu Liu ${ }^{\mathrm{b}}$, Bochao Zhao ${ }^{\mathrm{c}}$, Huanyan $\mathrm{Xu}^{\mathrm{d}}$ \\ Harbin University of Science and Technology, Harbin, China

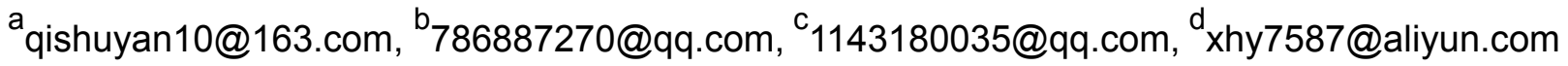

Keywords: Molybdenum diselenide, synthesis, application

\begin{abstract}
The structure, properties and functions of molybdenum diselenide are described briefly.The present situation of the synthesis of molybdenum diselenide is surveyed on the basis of the introduction of chemical, restacking and physical methods. The application of molybdenum diselenide in solar cells, lubricating oil, field-effect transistor eta. is introduced in details. The advantages and disadvantages of various methods were evaluated, with the development tendency of preparation technique of nanoscaled molybdenum diselenide shown and it was emphasized that more attention should be paid on the combinations of preparation methods of nanocomposites.
\end{abstract}

\section{Introduction}

Transition metal dichalcogenide (TMDs) are characterized by a $\mathrm{MX}_{2}$ formula, where $\mathrm{M}$ stands for a transition metal(Mo, W) and $\mathrm{X}$ stands for a chalcogen (Se, S, or Te). ${ }^{[1-2]}$ The layer type structure compounds transition metal dichalcogenides have been paid much attention in the last ten years because of their application in photo-voltaic、 photoelectrocatalytic solar energy converters、 electrocatalyst、solid lubricants, etc. ${ }^{[3]}$ Molybdenum diselenide $\left(\mathrm{MoSe}_{2}\right)$ is a member of transition metal dichalcogenides family. $\mathrm{MoSe}_{2}$ not only has all the characteristics of transition metal dichalcogenides described above. while compared with other disulfide, $\mathrm{MoSe}_{2}$ also has a back gate effect, solid lubrication properties etc. The superior performance of $\mathrm{MoSe}_{2}$ draw much attention of researchers in these years.

\section{Structure}

$\mathrm{MoSe}_{2}$ is grayish-black covalent compound with lamellar, close-packed hexagonal and layered structure that is similar to a sandwich. The atoms are held by covalent bounds while adjacent layers forming each other are Van der Waals forces.

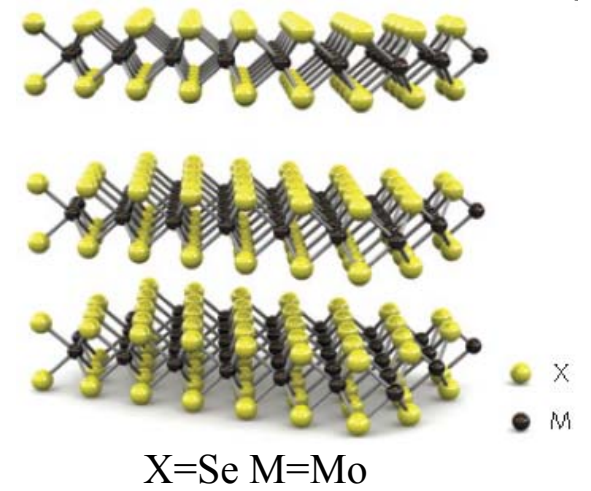

Figure 1. Structure of $\mathrm{MoSe}_{2}$

\section{2. preparation}

Nowadays, there have been a lot of methods for preparing $\mathrm{MoSe}_{2}$ at home and abroad. Generally, they can be divided into chemical method, physical method and restacking method. 
The substrate surface that is activated is immersed in the deposition solution without applied electric field and other energy. Thin films are deposited on the substrate surface through controlling complexation of reagents and chemical reaction at ordinary pressure and low temperature. Generally, the reaction processes in alkalescent condition. D. J.Sathe ${ }^{[4]}$ prepare $\mathrm{MoSe}_{2}$ with the precursor solution containing ammonium molybdate, sodium selenosulphite with hydrazine hydrate as a reducing agent. Optical properties indicate that the band gap energy of direct band gap is nearly $1.43 \mathrm{eV}$ and specific electrical conductivity is in the order of $10^{-5}(\Omega \mathrm{cm})^{-1}$. Huaqiang Shi eta. ${ }^{[5]}$ have reported a chemical reaction where di-(2,4,4-trimethylpentyl) dithiophosphinic acid extractant is used as reducing agent, home-made NaHSe is used as Se source, to synthesis flake-like $\mathrm{MoSe}_{2}$ nanomaterials. The measurements of XRD, TEM, EDXS indicates that flake-like $\mathrm{MoSe}_{2}$ with a diameter of about $70 \mathrm{~nm}$ was obtained.

\subsubsection{Chemical vapor deposition method}

So far, the chemical vapor deposition method is the most superior in so many methods for preparing transition metal dichalcogenides. Because the surface of samples may be damaged and the crystallinity of sample may be reduced, which is obtained by plasma, laser, or annealing process. Jonathan. Shaw ${ }^{[6]}$ used Se and $\mathrm{MoO}_{3}$ as the chemical vapor supplement to prepare optically distinguishable single-layer and multi-layer nanosheets on the typically triangular shaped domains with edge lengths around 30 um of $300 \mathrm{~nm} \mathrm{SiO}_{2} / \mathrm{Si}$ substrates.

\subsection{3 chemical vapor transport(CVT) method}

$\mathrm{MoSe}_{2}$ polycrystalline powders obtained through chemical vapor transport have been studied for many years, which carbon assisted mass transport enhancement effect is used to obtain the target crystal. S.Y.Hu ${ }^{[7]}$ utilized CVT process with $\mathrm{Br}_{2}$ as a transporting agent grow large size $\mathrm{MoSe}_{2}$ single crystals. The maximum size are about $10 \mathrm{~mm} \times 10 \mathrm{~mm}$ in surface area and $2 \mathrm{~mm}$ in thickness.Alberto ubaldini ${ }^{[8]}$ used four different transport agents: $\mathrm{I}_{2}, \mathrm{NH}_{4} \mathrm{Cl}, \mathrm{NH}_{4} \mathrm{Br}$ and $\mathrm{NH}_{4} \mathrm{I}$ for preparing single crystals of $\mathrm{MoSe}_{2}$. A ratio between $\mathrm{I}_{2}$ and $\mathrm{Mo}$ as high as 0.1 and 0.2 for other transport agents was used.

\subsection{Restacking single layer of MoSe2 method}

Object substance of restaking method is a lot including $\mathrm{Co}^{2+}, \mathrm{R}_{4} \mathrm{~N}^{+[9]}, 1,10$ - phenanthroline ${ }^{[10]}$, $\mathrm{Fe}(\mathrm{III})$-porphyrin ${ }^{[11]}$, eta in restacking method.

The properties of $\mathrm{MoSe}_{2}$-IC show undergone tremendous change in optical, electrical, magnetic, catalytic, and other functions. W.Sienicki ${ }^{[12]}$ have reported that the intercalated $\mathrm{MoSe}_{2}$ was obtained in the form of polycrystalline thin films $\mathrm{M}_{0.5} \mathrm{MoSe}_{2}$, where $\mathrm{M}$ stands for $\mathrm{Ga}$, In or $\mathrm{Tl}$.

\section{3 physical method}

Physical method is described that $\mathrm{MoSe}_{2}$ is produced through mechanical grinding, high energy physics and other means to achieve the purpose. J.H.Zhan ${ }^{[13]}$ used a stoichiometric amount of elemental molybdenum and selenium in a sealed evacuated tube at a temperature of at least $900^{\circ} \mathrm{C}$ for several days to synthesize crystalline molybdenum diselenide. Crystalline was yielded through a metathetical reaction between high-valent molybdenum halides and alkali-metal selenides. J. C. Bernede ${ }^{[14]}$ heated molybdenum powders and selenium powders under dynamic vacuum at $680 \mathrm{~K}$ for 24h. Stoichiometric layers $\mathrm{MoSe}_{2}$ have been obtained and are hexagonal structure through testing.

\section{Application}

\subsection{Application for solar cells}

The solar cell contains a first electrode layer and a second electrode layer. In the p-type semiconductor layer, a first region at the n-type semiconductor layer side and a second region at the first electrode layer side are different from each other. Semiconductor materials $\mathrm{MoSe}_{2}$ meet all the requirement for electrode of solar ceels. In addition, the prevention of electrolyte corrosion is main advantages of $\mathrm{MoSe}_{2}$ because non-bonding d-d-orbitals of $\mathrm{Mo}$ atoms is involved in the phototransition. Kristl $\mathrm{M}^{[15]}$ make the product whose configuration of fabricated cell is $\mathrm{n}-\mathrm{MoSe}_{2} \mid$ $\mathrm{NaI}(2 \mathrm{M})+\mathrm{I}_{2}(1 \mathrm{M}) \mid \mathrm{C}$. The fill factor and efficiency of the cell were found to be 34.22 and $1.01 \%$ respectively. 


\subsection{Application for lubricating oil}

MoSe2 have a crystal structure, which is similar to graphite and whose layers are held by van der waals forces. MoSe2 slip easily between the layers. Golub A S[16] use chemical vapor deposition method for preparing IF-MoSe2 nano materials and the lamellar MoS2. MoSe2 and MoS2 are dispersed by Span 80 dispersion and then put in UMT-2 friction test machine for friction test. The results show that IF-MoSe2 is a much better antiwear and friction-reducing additive in liquid paraffin than MoS2. Powell A Vi[17] use X-ray diffraction, scanning electron microscopy and transmission electron microscopy to characterize MoSe2. Friction test is operated to investigate the tribological properties of the $150 \mathrm{~nm}$ lubricating oil added with $\mathrm{MoSe} 2$ nanoflakes. The results indicates that the friction coeficient of the basic oil added with MoSe2 is higher than the basic oil.

\subsection{Application for field-effect transistor}

Field-effect transistors having an extremely high input impedance, low noise, high limited frequency, low power consumption, simple manufacturing process, good temperature characteristics and other good characteristics, are widely used in various amplification circuits, digital circuits and microwave circuits. Nakagaki S[18] used ultra-thin, mechanically exfoliated MoSe2 flakes to fabricate the back-gated field-effect transistors. The MoSe2 field-effect transistors with On/Off ratios lager than 106 are n-type and have a high gate modulation. A discovering explained by the presence of Schottky barriers at the metal contact/MoSe2 interface indicates that FETs have asymmetric characteristics on swapping the source and drain. The intrinsic conductivity and mobility of MoSe2 as a function of gate bias are measured through using four-point and back-gated devices. FETs having a room temperature mobility of $\sim 50 \mathrm{~cm} 2 / \mathrm{V}$ indicates that a strong temperature dependence, suggesting phonons are a dominant scattering mechanism.

\section{Conclusion and prospect}

In summary, the methods described above have advantages and disadvantages. MoSe 2 meet different functional requirements can be prepared by chemical methods . However, there are also disadvantages for chemical methods, such as the high requirement of raw materials, long process flow, and high production costs. The defects of chemical methods can be remedied by physical methods and the natural lattice of $\mathrm{MoSe} 2$ will be not destroyed. But the machine requirement of physical methods is higher, the method is not flexible enough, and products obtained are singular. Thus the development direction of preparation of $\mathrm{MoSe} 2$ diversify into a new process that the chemical method and physical method are combined each other.

Compared with the most common photo-catalytic materials $\mathrm{TiO} 2$, the band gap of $\mathrm{MoSe} 2$ is narrower, which is about $1.43 \mathrm{ev}$ and make it absorb visible light and near-infrared light. This is a premise that $\mathrm{MoSe} 2$ become photo-catalytic materials that is widely used.

Currently, the research of the preparation and application of MoSe 2 is still in the initial stage, which a large area of production and application can not be realized. Researchers at home and abroad should continue to work hard.

\section{Acknowledgments}

We thank the National Natural Science Foundation of China (51404083) and Youth Science Fund Project of HeiLongJiang Province,China (QC2012C084) for financial support.

\section{References}

[1]Yoffe A D. Low-dimensional systems: quantum size effects and electronic properties of semiconductor microcrystallites (zero-dimensional systems) and some quasi-two-dimensional systems.[J]. Advances in Physics, 1993, 42(2): 173-262.

[2]Ataca C, Ciraci S. Dissociation of $\mathrm{H}_{2} \mathrm{O}$ at the vacancies of single-layer $\mathrm{MoS}_{2}$. [J]. Physical Review B, 2012, 85(19): 195410. 
[3]Frindt R F, Yoffe A D. Physical properties of layer structures: optical properties and photoconductivity of thin crystals of molybdenum disulphide.[J]. Proceedings of the Royal Society of London. Series A. Mathematical and Physical Sciences, 1963, 273(1352): 69-83.

[4]Sathe D J, Chate P A, Hankare P P, et al. A novel route for synthesis, characterization of molybdenum diselenide thin films and their photovoltaic applications[J]. Journal of Materials Science: Materials in Electronics, 2013, 24(2): 438-442.

[5]Shi H, Zhou X, Lin Y, et al. Synthesis of $\mathrm{MoSe}_{2}$ nano-flakes modified with dithiophosphinic acid extractant at low temperature[J]. Materials Letters, 2008, 62(21): 3649-3651.

[6]Shaw J C, Zhou H, Chen Y, et al. Chemical vapor deposition growth of monolayer $\mathrm{MoSe}_{2}$ nanosheets[J]. Nano Research, 2014, 7(4): 1-7.

[7]Hu S Y, Liang C H, Tiong K K, et al. Effect of Re dopant on the electrical and optical properties of $\mathrm{MoSe}_{2}$ single crystals[J]. Journal of alloys and compounds, 2007, 442(1): 249-251.

[8]Ubaldini A, Jacimovic J, Ubrig N, et al. Chloride-Driven Chemical Vapor Transport Method for Crystal Growth of Transition Metal Dichalcogenides[J]. Crystal Growth \& Design, 2013, 13(10): 4453-4459.

[9]Pol V G, Pol S V, George P P, et al. Combining $\mathrm{MoS}_{2}$ or $\mathrm{MoSe}_{2}$ nanoflakes with carbon by reacting $\mathrm{Mo}(\mathrm{CO})_{6}$ with $\mathrm{S}$ or Se under their autogenic pressure at elevated temperature[J]. Journal of Materials Science, 2008, 43(6): 1966-1973.

[10]Srivastava S K, Avasthi B N. Synthesis and characterization of indium intercalation compounds of molybdenum diselenide, In $x \operatorname{MoSe}_{2}(0 \leq \mathrm{x} \leq 1)[\mathrm{J}]$. Journal of Materials Science, 1989, 24(6): 1919-1924.

[11] Xu H, Aylott J W, Kopelman R, et al. A real-time ratiometric method for the determination of molecular oxygen inside living cells using sol-gel-based spherical optical nanosensors with applications to rat $\mathrm{C}_{6}$ glioma[J]. Analytical chemistry, 2001, 73(17): 4124-4133.

[12]Agarwal M K, Patel P D, Gupta S K. Effect of doping $\mathrm{MoSe}_{2}$ single crystals with rhenium[J]. Journal of crystal growth, 1993, 129(3): 559-562.

[13]Zhan J H, Zhang Z D, Qian X F, et al. Synthesis of $\mathrm{MoSe}_{2}$ nanocrystallites by a solvothermal conversion from $\mathrm{MoO}_{3}[\mathrm{~J}]$. Materials research bulletin, 1999, 34(4): 497-501.

[14]Bernede J C, Pouzet J, Alaoui Z K. Preparation and characterization of molybdenum diselenide thin films[J]. Applied Physics A, 1990, 51(2): 155-159.

[15]Kristl M, Drofenik M. Synthesis of nanocrystalline $\mathrm{MoSe}_{2}$ by sonochemical reaction of Se with Mo $(\mathrm{CO})_{6}[\mathrm{~J}]$. Inorganic chemistry communications, 2003, 6(1): 68-70.

[16]Golub A S, Zaikovskii V I, Lenenko N D, et al. Intercalation of molybdenum disulfide with chlorobis (phenanthroline) copper (II)[J]. Russian chemical bulletin, 2005, 54(12): 2738-2745.

[17]Powell A V, Kosidowski L, McDowall A. Novel intercalation compounds of $\operatorname{MoS}_{2}[\mathrm{~J}]$. Molecular Crystals and Liquid Crystals, 2000, 341(2): 125-130.

[18]Nakagaki S, Mangrich A S, Wypych F. A cationic iron (III) porphyrin encapsulated between the layered structure of $\mathrm{MoS}_{2}$. A new approach to the synthesis of an Fe-Mo-S system[J]. Inorganica chimica acta, 1997, 254(2): 213-217. 